Supporting Information:

\title{
Universal Strength Scaling in Carbon Nanotube Bundles with Frictional Load Transfer
}

\author{
Nitant Gupta, ${ }^{\dagger}$ John M. Alred, ${ }^{\dagger, \dagger}$ Evgeni S. Penev, ${ }^{\dagger}$ and Boris I. Yakobson ${ }^{*, \dagger}$ \\ $\dagger$ Department of Materials Science and NanoEngineering, Rice University, Houston, Texas \\ 77005, United States \\ $\ddagger$ Current address: NASA Jet Propulsion Laboratory, Pasadena, California 91109, United \\ States
}

E-mail: biy@rice.edu 


\section{CONTENTS}

S1. CNT coarse-grained models: brief overview

S2. Model description

S3. Parameterization of friction at the CNT-CNT interface through granular potential

S4. Modeling different constitutive behavior of the CNT-CNT interfaces

S5. Choice of $r_{0}$ and its effect on load transfer

3

S6. Analytical derivations of load transfer across a CNT-CNT interface

S6.1. Elastic interface

S6.2. Plastic interface

S7. Implementation and tensile loading simulation in LAMMPS

References

\section{S1. CNT COARSE-GRAINED MODELS: BRIEF OVERVIEW}

Pseudo-molecular coarse-grained representations have long been in use especially in modeling biomolecular system, like proteins [1] and lipid membranes [2]. Over the past decade roughly, CG models have also been introduced for nanocarbon based forms, from pristine CNTs, to flattened, functionalized CNTs and eventually specific CNT-polymer composites. Table S2 summarizes some more recent works that have focused on related systems. Note that frictional force explicitly enters only the DPD (dissipative particle dynamics) scheme [3-5], however it is arbitrarily chosen, merely as a means of effective thermostating.

\section{S2. MODEL DESCRIPTION}

The coarse grain model uses linear harmonic and harmonic angular terms for the interactions of beads of the same CNTs.

$$
E_{r}=k\left(r-r_{0}\right)^{2}, \quad E_{\theta}=k_{\theta}\left(\theta-\theta_{0}\right)^{2}
$$

The energy of a linear harmonic interaction, $E_{r}$, is characterized by $k_{r}$, the linear stiffness, $r$, the distance between two adjacent beads of the same CNT, and $r_{0}$, the equilibrium separation distance of two adjacent beads of the same CNTs. Similarly, energy of an angular harmonic interaction, $E_{\theta}$, is characterized by $k_{\theta}$, the angular stiffness, $\theta$, the angle between three adjacent beads of the same CNT, and $\theta_{0}=\pi$, the equilibrium angle of three beads of the same CNTs.
For beads of different CNTs, the interaction is a 12-6 Lennard-Jones (LJ) potential with shifted argument,
1

1

Collectively, for a given CNT diameter $d$ and chosen $r_{0}$, the $\left\{k, k_{\theta}, \epsilon, \sigma_{0}\right\}$ parameters are fit to reproduce the elastic modulus, bending stiffness, and dispersion interaction of CNTs. The quality of the CG procedure/model largely depends on the choice of $r_{0}$, which is discussed in detail in Sec. S5. The parameterization of the CG model is summarized in Table S1.

TABLE S1. CG model parameters.

\begin{tabular}{ccccc}
\hline \hline$r_{0}$ & $k_{r}$ & $k_{\theta}$ & $\epsilon$ & $\sigma_{0}$ \\
$\AA$ & $\mathrm{kcal} / \mathrm{mol}^{2}$ & $\mathrm{kcal} / \mathrm{mol} \mathrm{rad}^{2}$ & $\mathrm{kcal} / \mathrm{mol}$ & $\AA$ \\
\hline 2 & 2500 & 35750 & 9.323 & 0.4205 \\
\hline \hline
\end{tabular}

$$
E_{\mathrm{LJ}}(r ; d)=4 \epsilon\left[\left(\frac{\sigma_{0}}{r-d}\right)^{12}-\left(\frac{\sigma_{0}}{r-d}\right)^{6}\right]
$$

\section{S3. PARAMETERIZATION OF FRICTION AT THE CNT-CNT INTERFACE THROUGH GRANULAR POTENTIAL}

To mimic cross-link induced friction, a granular-type potential was overlayed in the CG model. This potential is available as "pair_style gran/hooke/history" in LAMMPS, and derived from Ref. 19. This is a contact based potential, therefore, the particle centers must lie within a certain cut-off distance $D$. This is achieved by setting the diameter of the granular beads larger than inter-tube distance, i.e.,

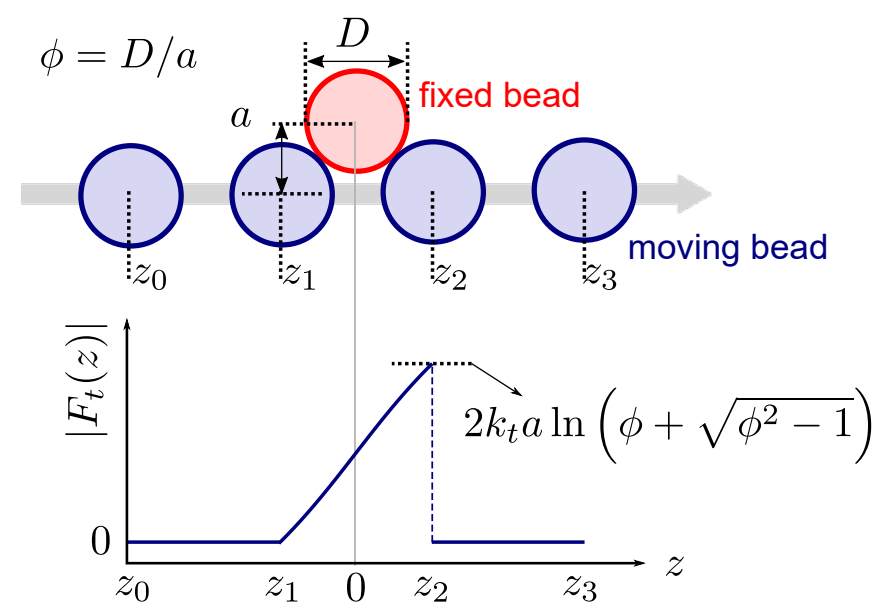

FIG. S1. Parameterization of granular friction. The tangential friction force as experienced by a moving bead as it passes by another fixed bead. Until the beads come into contact, no force is exchanged. As soon as the bead make contact the tangential force increases as described by equation (S4), and reaches a maximum force with magnitude $2 k_{t} a \ln \phi+\sqrt{\phi^{2}-1}$, upon which it breaks. The curve described by equation (S4) is very close to a straight line, and thus is used to approximate the force due to a recoverable crosslink. 
TABLE S2. Recent pseudo-molecular CG models of CNT-based systems.

\begin{tabular}{|c|c|c|c|}
\hline System & Representation & Interactions & Sources \\
\hline $\begin{array}{l}\text { CNT buckypaper, bundles, } \\
\text { works, films, yarns }\end{array}$ & net- bead-spring & bonded (bonds, angles): harmonic; nonbonded (vdW): Lennard-Jones & {$[6-10]$} \\
\hline CNT-polymer composites & bead-spring & Same as above, including bonded torsional term & [12] \\
\hline Flattened CNTs & bead-spring & bonded (bonds, angles): harmonic, nonbonded (vdW): Lennard-Jones & [13] \\
\hline CNT assemblies & $\begin{array}{l}\text { spherical or cylindri- } \\
\text { cal elements }\end{array}$ & 'Distinct element method', parallel bonded contacts & {$[14,15]$} \\
\hline CNT bundles/assemblies & $\begin{array}{l}\text { cylindrical } \\
\text { "CNT segments" }\end{array}$ & mesoscale potential; vdW: Lennard-Jones & {$[16-18]$} \\
\hline $\begin{array}{l}\text { CNTs, CNT-polycarbonate } \\
\text { posites }\end{array}$ & com- bead-spring & $\begin{array}{l}\text { Dissipative particle dynamics (DPD) forces including nonbonded } \\
\text { (vdW): Lennard-Jones }\end{array}$ & {$[3-5]$} \\
\hline
\end{tabular}

$D>a$. Briefly, the interaction between two such granular beads is thus written as:

$$
F_{n}=k_{n} \delta, \quad F_{t}=-k_{t} u_{t}
$$

Here, $k_{n}$ and $k_{t}$ are normal and tangential stiffness parameters for these interactions. The normal force thus depends on the overlap distance $\delta$, while the tangential force depends on the accumulated displacement $u_{t}$ during the time of contact. Thus, $u_{t}=\int v d t$, where $v$ is the relative velocity of the beads. Here we neglect the dynamic terms in the normal and tangential forces (by setting the coefficients to zero in the LAMMPS implementation; see below and also Sec. S7), as the simulations are performed in a quasi-static manner.

This potential is incorporated in our model by deriving the analytical relations for the forces on a moving bead as it passes by a stationary bead (Fig. S1). Since these beads will almost always be part of two parallel tubes, their trajectories are two parallel straight lines separated by distance $a$. The four position markers $\left(z_{0}<z_{1}<z_{2}<z_{3}\right)$ in Fig. $\mathrm{S} 1$ describe the relative position of the moving bead as it approaches the fixed bead with $z_{0}, z_{1}$ : indicating that the beads are well-separated, $z_{1}, z_{2}$ : beads are touching. The coordinate of the fixed bead is 0 , and thus it is easy to see that $z_{2}=-z_{1}=\sqrt{D^{2}-a^{2}}$. The two beads thus overlap when $z_{1}<z<z_{2}$, and in this interval, using the equations as described in Ref. 19, one can obtain the normal and tangential forces as:

$$
\begin{aligned}
& F_{n}(z)=k_{n}\left(D-\sqrt{a^{2}+z^{2}}\right), \\
& F_{t}(z)=-k_{t} a \ln \left(\frac{z+\sqrt{z^{2}+a^{2}}}{D-\sqrt{D^{2}-a^{2}}}\right) .
\end{aligned}
$$

In the modeling of crosslink induced friction, all forces normal to the CNT interfaces are ignored, since tubes are in a hexagonal close packed structure, which essentially keeps them in place. Moreover, unlike conventional (macroscopic) friction, the crosslink induced friction is not directly impacted by normal reaction between the tubes, since if the tubes are pressed against each other the crosslinks may simply become slack instead of producing any normal force. Thus ideally $k_{n}$ should be set to zero. However, in the LAMMPS imple- mentation, the normal force $F_{n}$ directly controls $F_{t}$ by setting $F_{t} \leqslant \mu F_{n}$, where $\mu$ is a parameter (akin to coefficient of friction). To counter these issues, we supplemented our own pair-wise potential which solely subtracts $F_{n}$, such that total normal force between two granular spheres is always zero, regardless of the value of $k_{n}$, and only remaining force is $F_{t}$. This term could be conveniently added as an additional term to the existing pair-wise potential that we use for $\mathrm{CG}$ interactions, namely "pair_style $1 j$ /expand". Therefore, the available source file pair_lj_expand.cpp was modified to make a new pair-wise potential in pair_lj_expand_gran.cpp and accordingly its LAMMPS command was updated as: "pair_style lj/expand/gran"; see the representative LAMMPS script in Sec. S7. The source files, along with documentation for this potential are included in the supplementary files.

After elimination of the normal force $F_{n}(z)$, the tangential force $F_{t}(z)$ behaves like a piece-wise linear function (see Fig. S1). In a CG tube, an ensemble of beads will interact with beads of a neighboring tube, and thus all the individual interactions need to be summed up to obtain an effective friction force per length as:

$$
f=\frac{-2 k_{t} a^{2}}{r_{0}^{2}}\left[\ln \left(\phi+\sqrt{\phi^{2}-1}\right)\right]^{2}
$$

where, $\phi \equiv D / a$, is the factor by which the granular bead diameter should exceed the inter-tube distance, Fig. S1. Thus, by simply tuning $D, a, r_{0}, k_{t}$ one can control the value of $f$.

\section{S4. MODELING DIFFERENT CONSTITUTIVE BEHAVIOR OF THE CNT-CNT INTERFACES}

The CG implementation of friction can be additionally tuned to produce different types of constitutive behavior of the CNT-CNT interfaces. As mentioned in the main text, the recoverability, or "healing", of the crosslinks controls this behavior ranging from elasto-plastic to brittle. If only the granular potential is used, the constitutive behavior is seen to be exclusively elasto-plastic. This is evident from the plots in Fig. S2(a). The latter shows the friction force per unit length 

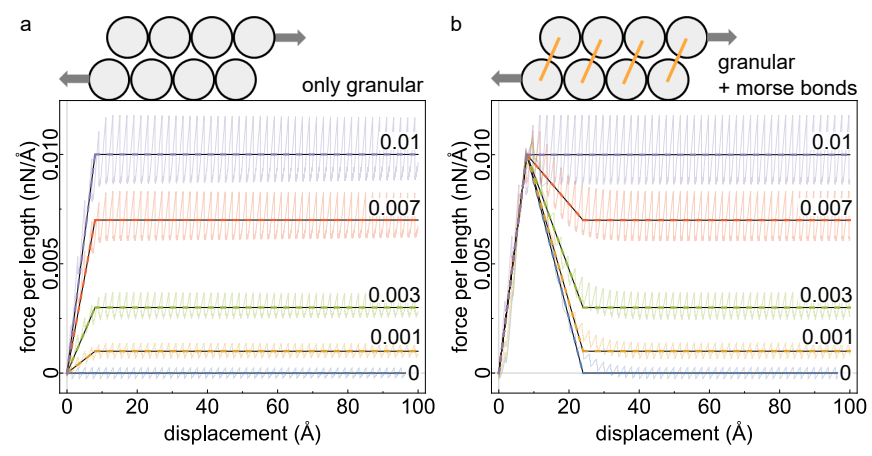

FIG. S2. Parameterization of constitutive behavior of the interface. Demonstration of frictional force per unit length between rigid CG tubes for (a) only using the granular potential with $f=0-0.01 \mathrm{nN} / \AA$, and (b) additionally using morse bonds, such that initial friction is always $0.01 \mathrm{nN} / \AA$.

measured between two rigid tubes (i.e., the beads of a given tube do not undergo relative displacement) for different values of $f$. Slight corrugation of the order of $r_{0}$ in the value of the force is seen due to coarse-graining itself, and thus piecewiselinear curves are fitted to represent the average force. At low displacement, the friction force increases linearly, and then becomes constant. Thus, if only the granular potential is used, the crosslinks are modeled as fully recoverable.

However, if crosslinks are either partially or completely nonrecoverable, the constitutive behavior should be transition towards a brittle interface. This can be modeled by incorporating breakable bonds such as Morse bonds (described by the Morse potential), together with the granular potential. Fig. S2(b) shows the combined effect of these potentials and accordingly the change in the constitutive relations.

\section{S5. CHOICE OF $r_{0}$ AND ITS EFFECT ON LOAD TRANSFER}

The inter-tube interaction is corrugated, $\sim V_{0}$, depending on the granularity as illustrated in Fig. S3. In CG models using $r_{0}=10 \AA$ and $\epsilon \simeq 0.65 \mathrm{eV},[6,7]$ such corrugation can reach $V_{0} / \epsilon \simeq 0.15$. The behavior of such CG model can be understood by considering it as a two-chain, finite-length Frenkel-Kontorova (FK) model, [20] and it has been shown that a corrugated interaction potential can produce a substantial interfacial force [21-23] $F \sim\left(k V_{0}\right)^{1 / 2}$. This force may be non-physical and undesirable for the purposes of modeling CNTs. In reality the interaction between two CNTs is corrugated at the atomic scale, but any difference in CNT chirality, or "helicity" $(n, m)$, or slight misalignment between tubes effectively eliminates this corrugation, which is not the case for the CG models. For $r_{0}=2 \AA$, the magnitude of the corrugation $V_{0}$ is negligible and consequently there is no artificially introduced friction. This value has been used for all simulations; cf. also Table. S1.

From the FK model, the approximate force required to nucleate a soliton is $\sim\left(k_{r} V_{0}\right)^{1 / 2}=12.3 \mathrm{nN}$, which is close to the load transfer saturation for a CG with $r_{0}=10 \AA$.
This is two orders of magnitude larger than the the capillary force caused by the vdW interaction between two pristine CNTs. This is due to the highly corrugated potential for $r_{0}=10 \AA$ which can be seen in Fig. S3(a). The CG model with $r_{0}=2 \AA$, as used in our parameterization, on the other hand, correctly reproduces the capillary force between two pristine CNTs. With negligible corrugation the load transfer between CNTs is simply the force required to create a new surface, which can be estimated as the tube-tube adhesion energy per unit length $F_{c} \simeq 0.14 \mathrm{nN}$; see also discussion in the main text.

Figure S3(b) shows the strain in both CNTs before the interface has yielded. The strain is constant throughout a majority of the central portion of the CNT, and only varies near the loaded and free ends of the CNT. This implies that load transfer only occurs at the ends of the CNT. This is in agreement the shear-lag model. [23-25] Figure S3(c) shows the strain distribution in the tube once interface yielding has occurred. The overall strain distribution looks similar except for distinct localized strain solitons. The tube with fixed end can be seen as a "substrate", which provides a deformable 1D potential, instead of the static periodic potential in the original FK model. The interface slip under tension occurs via nucleation and propagation of localized bond deformations, essentially pairs of tensile and compressive solitons, Figure S3 (c,d). The force in the tubes is highest at the loaded ends and because of this the solitons will be nucleated at the ends of the tubes.

The coarse-graining length and energy scales determine the width $w \sim r_{0}^{2}\left(k / V_{0}\right)^{1 / 2}$, and energy $\sim\left(k r_{0}^{2} V_{0}\right)^{1 / 2}$ of such solitons. For long tubes, $L \gg w$, slip can occur at threshold force $[21,23,26] \sim \sqrt{k V_{0}}$. The soliton yielding behavior is an artifact of the CG model using $r_{0}=10 \AA$ and its highly corrugated potential. In actuality CNTs do have a corrugated potential but the energy scale of the corrugation is so small that the soliton is completely delocalized. The CG model with $r_{0}=2 \AA$ does not exhibit this artificial yielding behavior and more accurately models realistic CNT. Figure S3(d) is a rendering of the topological soliton for $r_{0}=10 \AA$, with $w \sim 100 \mathrm{~nm}$. There are two types of interface alignments seen in this rendering: triangularly aligned (staggered, $\triangle$-interface), which is energetically more stable, and square aligned ( $\square$-interface). Where the soliton is localized there is one CG bead difference between the CNTs. This causes a gradual change from $\triangle$-interface to $\square$-interface back to $\triangle$-interface over a lengthscale $\sim w$.

\section{S6. ANALYTICAL DERIVATIONS OF LOAD TRANSFER ACROSS A CNT-CNT INTERFACE}

In the setup of Fig. S4 (and also Fig. 1 in the main text), two tubes of length $L$ each form a lateral interface with initial overlap $l=L$, and are symmetrically positioned across $z=$ 0 . Thus, for the lower tube the loaded end is at $z=-L / 2$ and the free end is at $z=L / 2$, and vice-versa for the upper tube. Since the loading is applied in a displacement controlled mode, as the tube ends are pulled, a load can be measured at the ends, and is written as $\sigma_{0} A_{0}$, with $A_{0}$ being the crosssectional area of the tube, and $\sigma_{0}$ is the stress at the ends. 

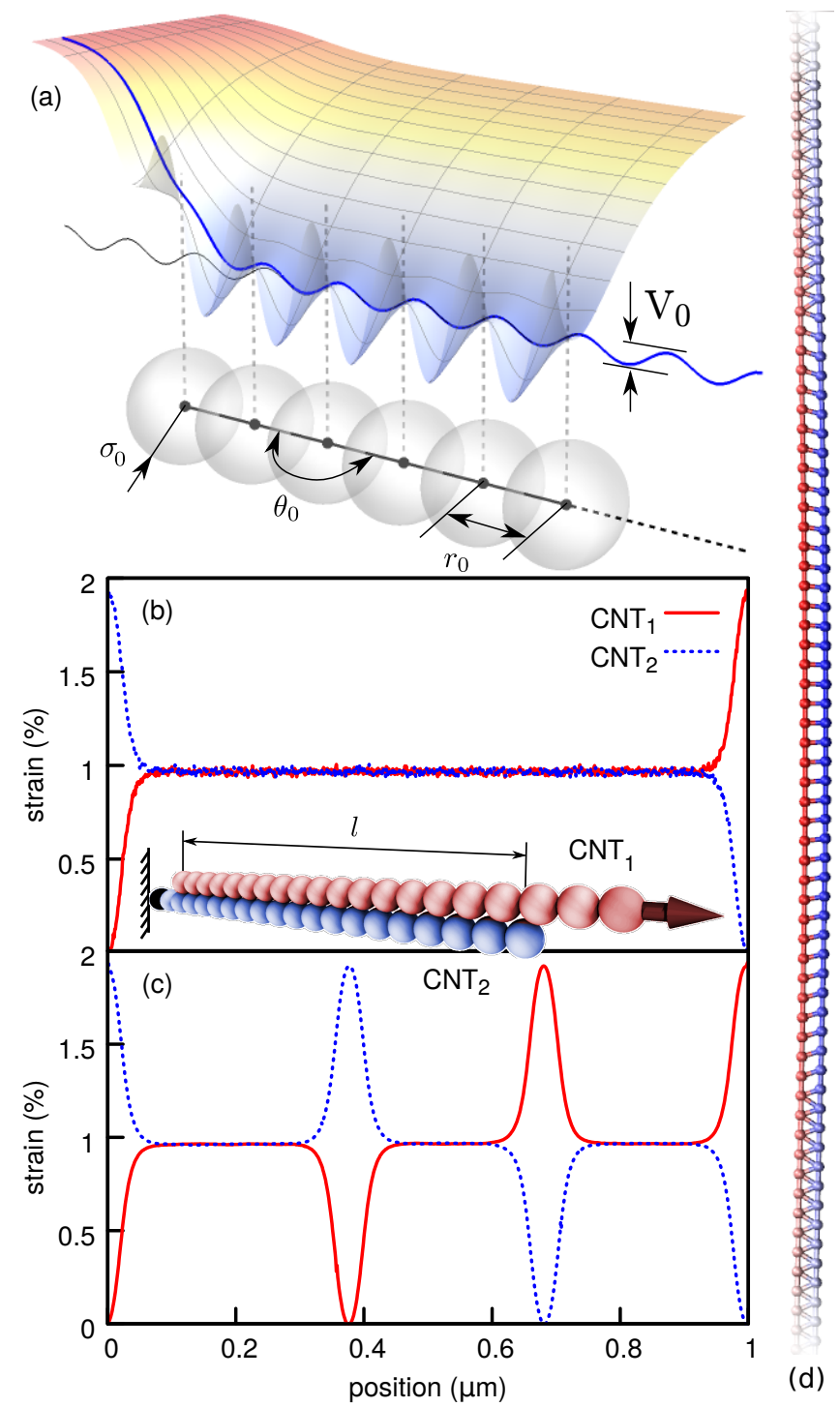

FIG. S3. Basic parameters of the CG model: equilibrium bond length $r_{0}$, angle $\theta_{0}$, and bead 'size' $\sigma_{0}$. The inter-tube interaction potential energy surface $V(\rho, z)$ is plotted along the axial and radial direction of the CNT. The blue line is a slice, $V(z)$, at a distance of $\rho_{0}=2^{1 / 6} \sigma_{0}$ from the tube axis $z$. Vertical dashed lines denote positions of the beads. The thin gray curve represents the function $\frac{1}{2} V_{0}[1-\cos (2 \pi z)]$. The strain before interface yielding occurs (b) and after yielding occurs (c) is plotted for a model with $r_{0}=10 \AA$. (d) "Topological soliton" identified as a local distortion of the staggered $\triangle$-interface into a $\square$-interface.

Two varying quantities, namely the stress along the tube $\sigma_{i}(z)$ and the frictional force per unit length along the interface $f_{i j}(z)$ (referred to as interface friction) are recognized. The expression for these quantities is derived below. Similar derivation can be found in Ref. 25, where the interface friction (force per length) is replaced by the shear stress (force per area) of the interface. Some key steps of this derivation are repeated here to keep the notations consistent, as well as to derive additional results that explain the stress-displacement curve in Fig. 1b (main text). Two sets of equations are ob- a

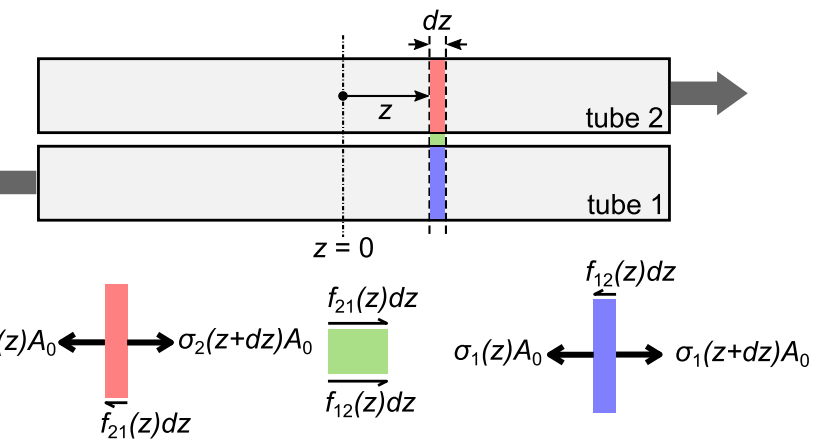

b

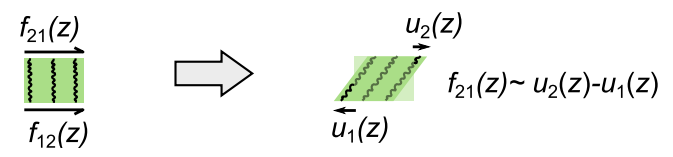

FIG. S4. Constitutive equations for a single interface. (a) Free-body diagrams for the force balance at a CNT-CNT interface. (b) Elastic response of the interface.

tained by balancing the forces at the cross-section of the nanotube, and also at the cross-section of the interface. At any tube cross-section the force balance is obtained from the stress in the tube and the friction from the interface. Also, at the interface, the forces are balanced between the two tubes due to the elasticity of the interface. This can be expressed in equations as:

$$
\begin{gathered}
\sigma_{1}^{\prime}(z) A_{0}=f_{12}(z)=-f_{21}(z)=-\sigma_{2}^{\prime}(z) A_{0} \\
f_{21}(z)=\frac{2 A_{0} Y}{\lambda^{2}}\left(u_{2}(z)-u_{1}(z)\right) \Longrightarrow \\
f_{21}^{\prime}(z)=\frac{2 A_{0}}{\lambda^{2}}\left(\sigma_{2}(z)-\sigma_{1}(z)\right) .
\end{gathered}
$$

Here $\lambda$ is the stress transfer length, [27] and can be obtained from the elastic properties of the interface. [25] Equations (S6) and (S7) can be combined to give the following differential equation:

$$
f_{21}^{\prime \prime}(z)=\frac{4}{\lambda^{2}} f_{21}(z) .
$$

For an interface which is completely elastic, the interface friction is unbounded and the solution to this equation can be written as:

$$
f_{21}(z)=f_{0} \cosh (z / \lambda) \operatorname{sech}(L / \lambda) .
$$

Here, $f_{0}$ is the frictional force per length at the end points of the interface. However, generally interface friction is bounded due to the finite elasticity of the interface, and upon reaching a maximum value should either become constant (i.e., elastoplastic behavior when the crosslinks are fully recoverable) or drop to zero (i.e., brittle behavior when the crosslinks are not recoverable). This maximum value is $f$ in the main text, and characterizes the density of crosslinks on the interface. Therefore, $f_{0} \leqslant f$.

Choosing the elasto-plastic behavior of the interface, the general solution to equation (S8) can be obtained in a piecewise manner. Along the length of the tube, three types of 


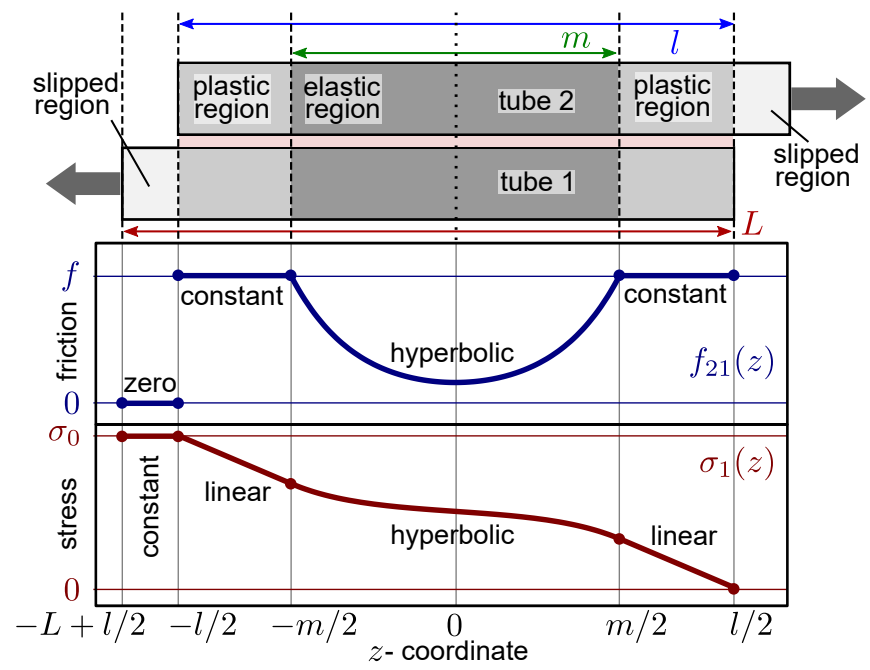

FIG. S5. Schematic of the CNT-CNT interface with different domains: elastic region (dark grey), plastic region (medium grey) and slipped region (light grey). In the elastic region, the crosslinks are still elastic, whereas in the plastic region the crosslinks are already participating in the fracturing-rebonding cycles (as explained in section 3). The interface friction $f_{21}(z)$ and stress $\sigma_{1}(z)$ are plotted and labeled with their functional forms. regions can be identified, as shown in Fig. S5 depending on whether the interface is locally (1) elastic, (2) plastic, or (3) absent. To clarify, the interface is absent when one of the tubes has already slipped away from the other tube as shown in Fig. S5. Here $l$ is the total overlap length between the two CNTs and $m$ is the length of the elastic region.

$$
f_{21}(z)=\left\{\begin{array}{l}
0, \quad-L+l / 2 \leqslant z \leqslant-l / 2 \\
f, \quad-l / 2 \leqslant z \leqslant-m / 2 \\
f \cosh (2 z / \lambda) \operatorname{sech}(m / \lambda), \quad-m / 2 \leqslant z \leqslant m / 2 \\
f, \quad m / 2 \leqslant z \leqslant l / 2
\end{array}\right.
$$

Integrating equation (S10), according to equation (S6), an expression for $\sigma_{1}(z)$ is obtained:

$\sigma_{1}(z)=-\frac{1}{A_{0}} \int_{z}^{L / 2} f_{21}\left(z^{\prime}\right) d z^{\prime}=\left\{\begin{array}{l}\frac{f}{A_{0}}(l-m+\lambda \tanh (m / \lambda)), \quad-L+l / 2 \leqslant z \leqslant-l / 2 \\ \frac{f}{A_{0}}(l / 2-m+\lambda \tanh (m / \lambda)-z), \quad-l / 2 \leqslant z \leqslant-m / 2 \\ \frac{f}{A_{0}}(l / 2-m / 2+(\lambda / 2) \tanh (m / \lambda)-(\lambda / 2) \sinh (2 z / \lambda) \operatorname{sech}(m / \lambda)), \quad-\frac{m}{2} \leqslant z \leqslant \frac{m}{2} \\ \frac{f}{A_{0}}(l / 2-z), \quad m / 2 \leqslant z \leqslant l / 2\end{array}\right.$

Furthermore, the deformed coordinate of the $\mathrm{CNT} Z_{1}(z)$ can also be obtained as:

$$
\begin{aligned}
Z_{1}(z)= & Z_{1}(0)+\int_{0}^{z}\left(1+\frac{\sigma_{1}\left(z^{\prime}\right)}{Y}\right) d z^{\prime} \\
= & \left\{\begin{array}{l}
z+\frac{f}{8 Y A_{0}}\left((l-m)(l+m+8 z)-2 \lambda^{2}+2(m+4 z) \lambda \tanh (m / \lambda)\right), \quad-L+l / 2 \leqslant z \leqslant l / 2 \\
z+\frac{f}{8 Y A_{0}}\left(-m^{2}-8 m z+4 z(l-z)-2 \lambda^{2}+2(m+4 z) \lambda \tanh (m / \lambda)\right), \quad-l / 2 \leqslant z \leqslant-m / 2 \\
z+\frac{f}{4 Y A_{0}}\left(-\lambda^{2} \cosh (2 z / \lambda) \operatorname{sech}(m / \lambda)+2 z(l-m+\lambda \tanh (m / \lambda))\right), \quad-m / 2 \leqslant z \leqslant m / 2 \\
z+\frac{f}{8 Y A_{0}}\left(-m^{2}+4 z(l-z)-2 \lambda^{2}+2 m \lambda \tanh (m / \lambda)\right), \quad m / 2 \leqslant z \leqslant l / 2
\end{array}\right.
\end{aligned}
$$

Both the piece-wise functions $f_{21}(z)$ and $\sigma_{1}(z)$ are shown in Fig. S5. Due to the symmetry of the setup, one can obtain corresponding expression of $\sigma_{2}(z)$ and $Z_{2}(z)$ as:

$$
\sigma_{2}(z)=\sigma_{1}(-z), \quad Z_{2}(z)=-Z_{1}(-z)
$$

With these solutions to the profile of $\sigma_{i}(z)$ and $Z_{i}(z)$, the stress at the loaded ends of each tube $\sigma_{0}$ and displacement between the loaded ends $u$ as functions of the two parameters $l$ and $m$, can thus be obtained as:

$$
\begin{aligned}
\sigma_{0}(l, m) & \equiv \sigma_{1}(-L+l / 2) \\
& =\frac{f}{A_{0}}(l-m+\lambda \tanh (m / \lambda)), \\
u(l, m) & \equiv Z_{2}(L-l / 2)-Z_{1}(-L+l / 2)-L \\
& =L-l+\frac{f}{A_{0} Y}\left[(l-m)\left(2 L-\frac{5 l+m}{4}\right)+\frac{\lambda^{2}}{2}\right. \\
& \left.+\left(2 L-l-\frac{m}{2}\right) \lambda \tanh (m / \lambda)\right] .
\end{aligned}
$$

These results can now be applied to explain the stress- 
displacement curve in Fig. 1b of the main text, where we refer to two distinct behaviors of the interface as elastic and plastic interfaces. These are explained in the following subsections.

\section{S6.1. Elastic interface}

During the displacement controlled loading of the interface, if the length of the elastic region (Fig. S5) is non-zero $(m \neq 0)$, then there is no net relative displacement between the two tubes, as the center-of-mass of the two tubes remain intact. In this situation, the two tubes undergo elastic loading, and maintain the same initial overlap, such that $l=L$. The interface behaves like a loaded spring, and is therefore called an elastic interface. We can evaluate equations (S14) and (S15) for this scenario as:

$$
\begin{aligned}
\sigma_{0}(L, m) & =\frac{f}{A_{0}}(L-m+\lambda \tanh (m / \lambda)) \\
u(L, m) & =\frac{f}{A_{0} Y}\left[(L-m)\left(\frac{3 L-m}{4}\right)+\frac{\lambda^{2}}{2}\right. \\
& \left.+\left(L-\frac{m}{2}\right) \lambda \tanh (m / \lambda)\right] .
\end{aligned}
$$

As can be noticed, a direct closed-form relationship between $u$ and $\sigma_{0}$ can not be obtained, and so only the numerical solution can be plotted as is done in Fig. 1b (main text). However, if $\lambda$ is small, that it can be neglected, the close-form expression can be approximated as:

$$
\sigma_{0}(u) \simeq \frac{f L}{A_{0}}\left(\sqrt{1+\frac{4 Y A_{0} u}{f L^{2}}}-1\right)
$$

If the value for $l=L$ is substituted in equations (S10) and (S11), the corresponding profiles of $f_{21}(z)$ and $\sigma_{1}(z)$, respec- tively can be obtained. This is what was plotted in Fig. 1c of the main text for the elastic interface.

\section{S6.2. Plastic interface}

When the length of the elastic region in Fig. S5 shrinks to zero $(m=0)$, the tubes undergo relative displacement, and the overlap length $l$ begins to shrink too $(l \leqslant L)$. In this situation, the interface yields, and is therefore called a plastic interface. We can evaluate equations (S14) and (S15) for this scenario as:

$$
\begin{aligned}
\sigma_{0}(l, 0) & =\frac{f}{A_{0}} l, \\
u(l, 0) & =L-l+\frac{f}{A_{0} Y}\left[l\left(2 L-\frac{5 l}{4}\right)+\frac{\lambda^{2}}{2}\right] .
\end{aligned}
$$

The closed-form expression of $\sigma_{0}(u)$ is indeed possible and is obtained as follows:

$$
\begin{aligned}
\sigma_{0}(u) & =\frac{4 f L}{5 A_{0}} \\
& +\frac{2 Y}{5}\left(\sqrt{1+\frac{4 f^{2} L^{2}}{Y^{2} A_{0}^{2}}+\frac{5 f^{2} \lambda^{2}}{2 Y^{2} A_{0}^{2}}+\frac{f(L-5 u)}{Y A_{0}}}-1\right) \\
& \simeq \frac{4 f L}{5 A_{0}}+\frac{2 Y}{5}\left(\sqrt{1+\frac{4 f^{2} L^{2}}{Y^{2} A_{0}^{2}}+\frac{f(L-5 u)}{Y A_{0}}}-1\right) .
\end{aligned}
$$

If the value for $m=0$ is substituted in equations (S10) and (S11), the corresponding profiles of $f_{21}(z)$ and $\sigma_{1}(z)$, respectively can be obtained. This is what was plotted in Fig. 1c of the main text for the plastic interface.

One can further show that eliminating $l, m$ from equations (S11) and (S15), the equation of the surface $\sigma(z, u)$ can be obtained that was plotted in the inset of Fig $1 \mathrm{~b}$ in the main text.

\section{S7. IMPLEMENTATION AND TENSILE LOADING SIMULATION IN LAMMPS}

Below we provide the actual script used to simulate the tensile loading whose $\sigma-\varepsilon$ curves are shown in Fig. 3b in the main text. This is a representative example of all simulations performed.

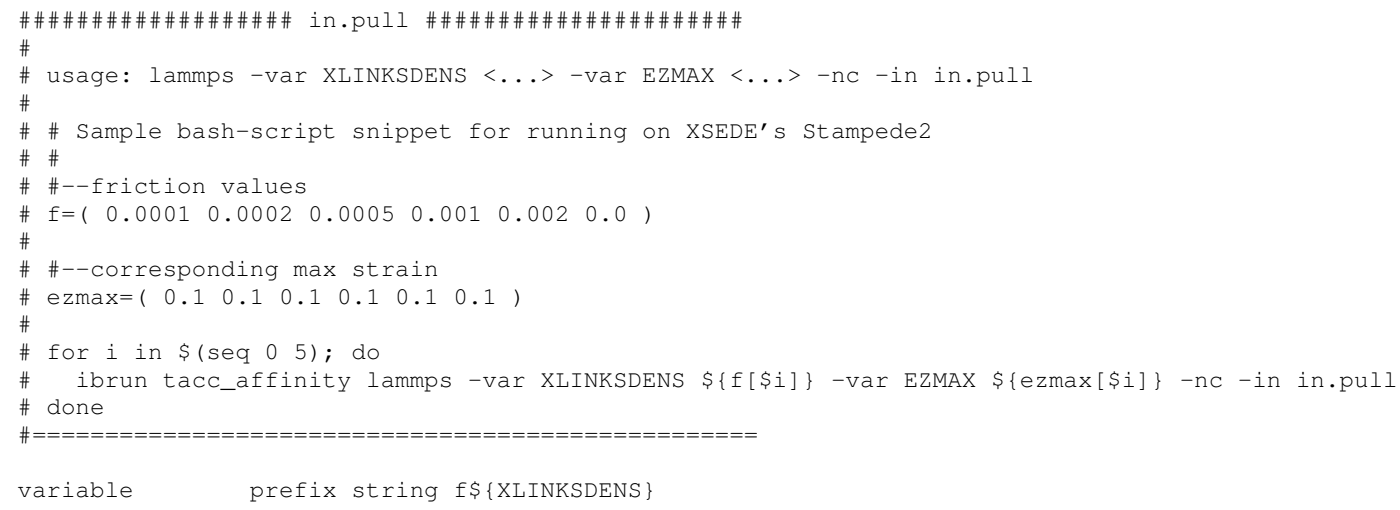




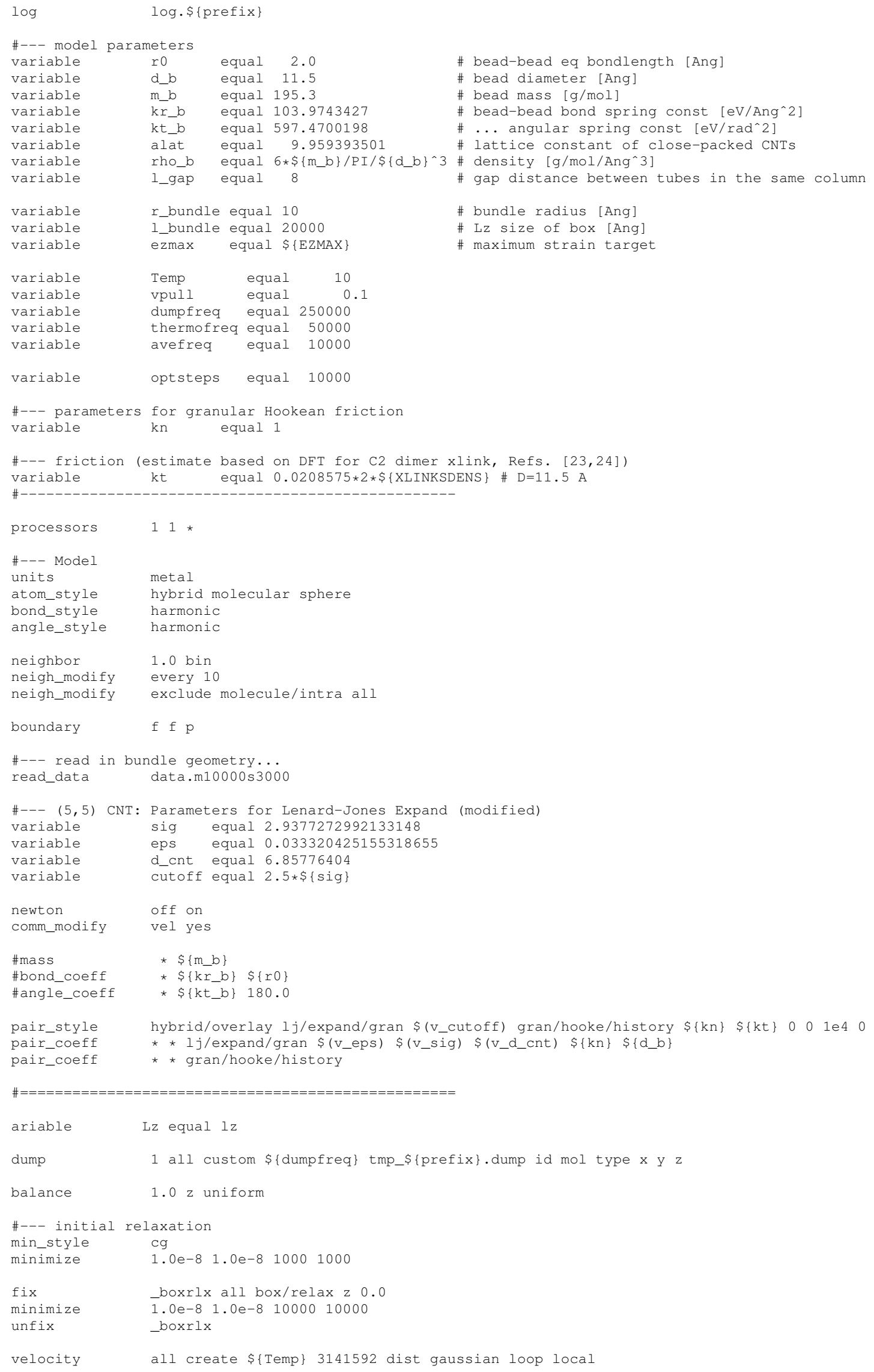




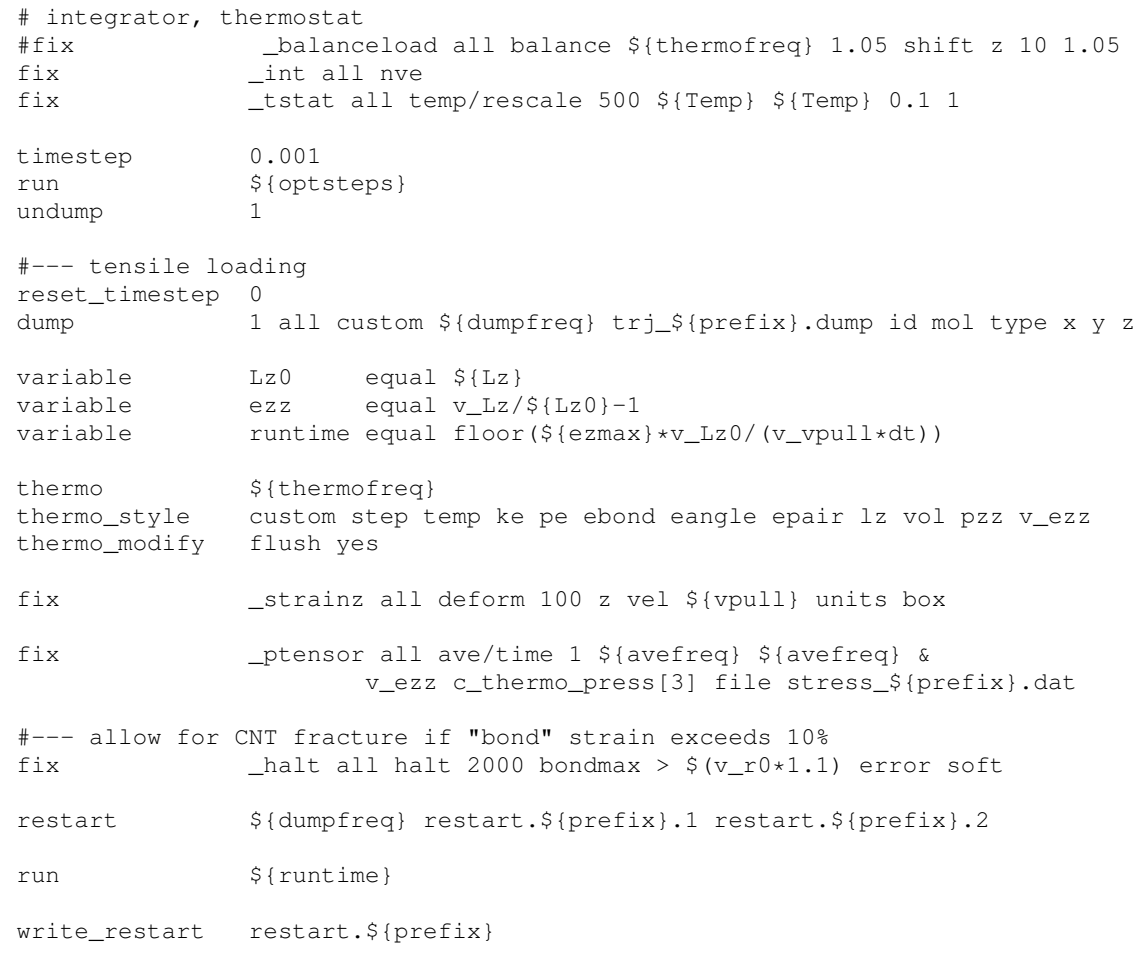

[1] Tozzini, V. Coarse-Grained Models for Proteins. Curr. Opin. Struct. Biol. 2005, 15, 144-150.

[2] Watson, M. C.; Penev, E. S.; Welch, P. M.; Brown, F. L. H. Thermal Fluctuations in Shape, Thickness, and Molecular Orientation in Lipid Bilayers. J. Chem. Phys. 2011, 135, 244701.

[3] Liba, O.; Kauzlarić, D.; Abrams, Z. R.; Hanein, Y.; Greiner, A.; Korvink, J. G. A Dissipative Particle Dynamics Model of Carbon Nanotubes. Mol. Simulation 2008, 34, 737-748.

[4] Wang, Y.-C.; Ju, S.-P.; Cheng, H.-Z.; Lu, J.-M.; Wang, H.-H. Modeling of Polyethylene and Functionalized CNT Composites: A Dissipative Particle Dynamics Study. J. Phys. Chem. C 2010, 114, 3376-3384.

[5] Wang, Y.-C.; Ju, S.-P.; Huang, T. J.; Wang, H.-H. Modeling of Polyethylene, Poly (L-lactide), and CNT Composites: A Dissipative Particle Dynamics Study. Nanoscale Res. Lett. 2011, 6, 433.

[6] Buehler, M. J. Mesoscale Modeling of Mechanics of Carbon Nanotubes: Self-Assembly, Self-Folding, and Fracture. $J$. Mater. Res. 2006, 21, 2855-2869.

[7] Cranford, S. W.; Buehler, M. J. In Silico Assembly and Nanomechanical Characterization of Carbon Nanotube Buckypaper. Nanotechnology 2010, 21, 265706.

[8] Wang, C.; Xie, B.; Liu, Y.; Xu, Z. Mechanotunable Microstructures of Carbon Nanotube Networks. ACS Macro Lett. 2012, 1, $1176-1179$.

[9] Zhao, J.; Jiang, J.-W.; Wang, L.; Guo, W.; Rabczuk, T. CoarseGrained Potentials of Single-Walled Carbon Nanotubes. J. Mech. Phys. Solids 2014, 71, 197-218.

[10] Lu, W.; Liu, X.; Li, Q.; Byun, J.-H.; Chou, T.-W. Mechanical Behavior and Structural Evolution of Carbon Nanotube Films and Fibers under Tension: A Coarse-Grained Molecular Dynamics Study. J. Appl. Mech. 2013, 80, 051015.

[11] Naraghi, M.; Bratzel, G. H.; Filleter, T.; An, Z.; Wei, X.; Nguyen, S. T.; Buehler, M. J.; Espinosa, H. D. Atomistic Investigation of Load Transfer between DWNT Bundles "Crosslinked" by PMMA Oligomers. Adv. Funct. Mater. 2013, 23, 1883-1892.

[12] Arash, B.; Park, H. S.; Rabczuk, T. Mechanical Properties of Carbon Nanotube Reinforced Polymer Nanocomposites: A Coarse-Grained Model. Composites Part B 2015, 80, 92-100.

[13] Liu, X.; Yang, Q.-S. Plastic Deformation and Failure Mechanisms of Collapsed-Carbon Nanotube Fibers by CoarseGrained Molecular Dynamic Simulations. Int. J. Plast. 2015, 64, 104-112.

[14] Ostanin, I.; Ballarini, R.; Potyondy, D.; Dumitrică, T. A Distinct Element Method for Large Scale Simulations of Carbon Nanotube Assemblies. J. Mech. Phys. Solids 2013, 61, 762-782.

[15] Ostanin, I.; Ballarini, R.; Dumitrică, T. Distinct Element Method for Multiscale Modeling of Cross-Linked Carbon Nanotube Bundles: From Soft to Strong Nanomaterials. J. Mater. Res. 2015, 30, 19-25.

[16] Volkov, A. N.; Zhigilei, L. V. Mesoscopic Interaction Potential for Carbon Nanotubes of Arbitrary Length and Orientation. $J$. Phys. Chem. C 2010, 114, 5513-5531.

[17] Volkov, A. N.; Banna, A. H. Mesoscopic Computational Model of Covalent Cross-Links and Mechanisms of Load Transfer in Cross-Linked Carbon Nanotube Films with Continuous Networks of Bundles. Comput. Mater. Sci. 2020, 176, 109410.

[18] Volkov, A. N.; Zhigilei, L. V. Structural Stability of Carbon Nanotube Films: The Role of Bending Buckling. ACS Nano 
2010, 4, 6187-6195.

[19] Silbert, L. E.; Ertaş, D.; Grest, G. S.; Halsey, T. C.; Levine, D.; Plimpton, S. J. Granular Flow down an Inclined Plane: Bagnold Scaling and Rheology. Phys. Rev. E 2001, 64, 051302.

[20] Vanossi, A.; Manini, N.; Urbakh, M.; Zapperi, S.; Tosatti, E. Colloquium: Modeling Friction: From Nanoscale to Mesoscale. Rev. Mod. Phys. 2013, 85, 529.

[21] Popov, A. M.; Lebedeva, I. V.; Knizhnik, A. A.; Lozovik, Y. E.; Potapkin, B. V. Commensurate-Incommensurate Phase Transition in Bilayer Graphene. Phys. Rev. B 2011, 84, 045404.

[22] Paci, J. T.; Furmanchuk, A.; Espinosa, H. D.; Schatz, G. C. Shear and Friction between Carbon Nanotubes in Bundles and Yarns. Nano Lett. 2014, 14, 6138-6147.

[23] Hammad, A.; Swinburne, T. D.; Hasan, H.; Del Rosso, S.; Iannucci, L.; Sutton, A. P. Theory of the Deformation of Aligned
Polyethylene. Proc. R. Soc. London A 2015, 471, 20150171.

[24] Chen, B.; Wu, P.; Gao, H. A Characteristic Length for Stress Transfer in the Nanostructure of Biological Composites. Compos. Sci. Technol. 2009, 69, 1160-1164.

[25] Wei, X.; Naraghi, M.; Espinosa, H. D. Optimal Length Scales Emerging from Shear Load Transfer in Natural Materials: Application to Carbon-Based Nanocomposite Design. ACS Nano 2012, 6, 2333-2344.

[26] Braun, O. M.; Kivshar, Y. S. Nonlinear Dynamics of the Frenkel-Kontorova Model. Phys. Rep. 1998, 306, 1-108.

[27] Hull, D.; Clyne, T. W. An Introduction to Composite Materials, 2nd ed.; Cambridge Solid State Science Series; Cambridge University Press: New York, 1996. 